\title{
Domino living donor liver transplantation of familial amyloid polyneuropathy patient - A case report -
}

Received July 13, 2020

Revised August 28, 2020

Accepted September 21, 2020

\section{Corresponding author}

Gaab Soo Kim, M.D., Ph.D.

Department of Anesthesiology and

Pain Medicine, Samsung Medical

Center, 81 Irwon-ro, Gangnam-gu,

Seoul 06351, Korea

Tel: 82-2-3410-0360

Fax: 82-2-3410-0361

E-mail: gskim@skku.edu

\section{Sungrok Cha', Jiwon Kim², Soo Joo Choi', and Gaab Soo Kim'}

Departments of ${ }^{1}$ Anesthesiology and Pain Medicine and ${ }^{2}$ Nursing, Samsung Medical Center, Seoul, Korea

Background: Familial amyloid polyneuropathy (FAP) is caused by a mutation in a gene transcribing transport protein produced mainly by the liver. Liver transplantation is required to stop FAP progression, but the pathology causes anesthetic management challenges.

Case: We report a case of domino living donor liver transplantation in a FAP patient. No intraoperative events occurred; however, during postoperative day 1 in the intensive care unit (ICU), the FAP patient underwent multiple cardiopulmonary resuscitation (CPR) sessions due to pulseless electrical activity following a sudden drop in blood pressure and ventricular tachycardia. Despite ICU management, the patient died after the third CPR session.

Conclusions: Various anesthetic management techniques should be considered for FAP patients. Anesthetic management was carefully assessed with the use of isoflurane, isoproterenol, and an external patch. The cause of deterioration in the ICU is unclear, but further investigation is needed to prevent and better manage postoperative morbidity and mortality.

Keywords: Autonomic denervation; Domino liver transplantation; Familial amyloid polyneuropathy; Isoproterenol; Living donor; Liver transplantation; Transthyretin.
Familial amyloid polyneuropathy (FAP) is a rare form of amyloidosis that is predominantly found in Portugal, Japan, and Scandinavia [1]. It is characterized by a progressive sensory-motor polyneuropathy with often severe autonomic neuropathy due to amyloid deposition [2]. Transthyretin (TTR) is a transport protein produced mainly by the liver that carries retinol-binding protein and thyroxine. Misfolding of TTR monomers causes aggregation which results in the formation of amyloid fibrils. TTR gene mutation makes the protein more prone to the dissociation to monomers from its natural tetramer forms; thus, formation and aggregation of amyloid fibrils are accelerated and its accumulation causes various impairments in the organ [3].
Thus, liver transplantation (LT) is required to stop its progression [4]. However, undergoing major surgical operations puts the patient at risk for various anesthetic complications due to various organ impairments caused by amyloid deposition.

Domino LT was first proposed in 1995 [5]. The liver of an FAP patient is fully functional, except for the production of mutant TTR. Since the clinical manifestation of amyloid deposition takes years to develop [6], it is reasonable to make use of the explanted liver for patients with hepatic failure or cancer who are in need of urgent LT.

Here, we present a series of living donor LTs (LDLTs; i.e., a domino LT), first performed in Korea. Institutional Review

This is an Open Access article distributed under the terms of the Creative Commons Attribution Non-Commercial License (http://creativecommons.org/licenses/by-nc/4.0) which permits unrestricted non-commercial use, distribution, and reproduction in any medium, provided the original work is properly cited.

Copyright (C) the Korean Society of Anesthesiologists, 2020 
Board approved the study (no. 2020-06-087) and waived the requirement for informed consent for all three patients.

\section{CASE REPORT}

Demographics of three patients are presented in Table 1, and timeline of operation is presented in Fig. 1.

\section{Case 1. First donor}

A 24-year-old man presented as a living donor for LT. Preoperative evaluation revealed a 2.5-pack-year smoking history without any other past medical history or abnormal

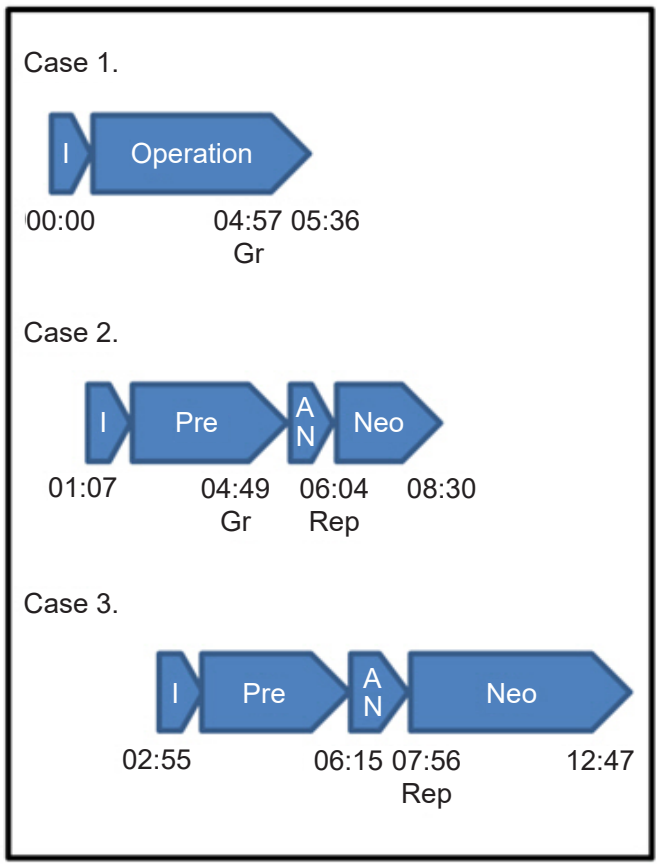

Fig. 1. Timeline of the three consecutive operations for the domino liver transplantation. All timelines are relative to the start of the operation for Case 1. I: induction, Gr: graft out, Pre: prehepatic stage, AN: anhepatic stage, Neo: neohepatic stage, Rep reperfusion. laboratory/imaging findings.

Spinal and general anesthesia was planned, and $2 \mathrm{mg}$ of midazolam was administered intravenously as a sedative. Spinal anesthesia was performed using 27 G Whitacre needle with a midline approach at L4-5. Morphine sulfate (400 $\mu \mathrm{g}$ ) was injected intrathecally without any acute complications. Intravenous thiopental (350 mg) was administered as an induction agent and vecuronium ( $8 \mathrm{mg}$ ) was used as a neuromuscular blocking agent. Continuous remifentanil infusion beginning at $0.2 \mu \mathrm{g} / \mathrm{kg} / \mathrm{min}$ was administered to control pain, as well as to manage discomfort caused by intubation. After mask ventilation with $100 \%$ oxygen and isoflurane for $3 \mathrm{~min}$, intubation was performed with ease. The arterial catheter was placed in the right radial artery for monitoring.

Anesthesia was maintained with isoflurane and continuous infusions of both vecuronium and remifentanil. Plasma solution $A^{\circledR}$ was used for hydration at a rate of $200 \mathrm{ml} / \mathrm{h}$. The Pringle maneuver was applied for $17 \mathrm{~min} 40 \mathrm{~s}$ without significant hemodynamic instability. Four hours after induction, 5,000 units of heparin were administered intravenously and the graft was harvested. Hydroxyethyl starch solution (500 ml) was infused following the harvest, and isoflurane was changed to desflurane. Pyridostigmine (15 $\mathrm{mg}$ ) paired with glycopyrrolate $(0.4 \mathrm{mg})$ was given as a reversal agent, and $25 \mathrm{mg}$ of pethidine was given as well. Total urine output was $130 \mathrm{ml}$ and the estimated blood loss (expressed as lost red blood cell [RBC] mass was) $463 \mathrm{ml}$ [7]. The donor was transferred to the post-anesthesia care unit after surgery and was discharged on the 10th postoperative day (POD) without any complications; no transfusions were required.

\section{Case 2. First recipient (second donor)}

A 59-year-old woman presented for living donor LT and

Table 1. Summary of Cases of Domino LT

\begin{tabular}{|c|c|c|c|}
\hline Variable & Case 1 & Case 2 & Case 3 \\
\hline Sex & $\mathrm{M}$ & $\mathrm{F}$ & $\mathrm{M}$ \\
\hline Age (yr) & 24 & 59 & 64 \\
\hline $\mathrm{BMI}\left(\mathrm{kg} / \mathrm{m}^{2}\right)$ & 21.67 & 20.88 & 28.96 \\
\hline Blood type & $\mathrm{B}+$ & $\mathrm{B}+$ & $\mathrm{B}+$ \\
\hline MELD & - & 8 & 9 \\
\hline Graft type & Right hemiliver & Extended right hemiliver & - \\
\hline Graft size (g) & 630 & 605 & - \\
\hline GRWR & - & 1.15 & 0.8 \\
\hline
\end{tabular}

BMI: body mass index, MELD: model for end-stage liver disease, GRWR: graft recipient weight ratio. 
as a liver donor for domino LT. She presented with symptoms of edema and paresthesia of both lower extremities 7 years ago and was found to have cardiomegaly on chest radiography. She was diagnosed with amyloidosis 1 year previously while undergoing a pericardial window operation for pericardial effusion.

Airway examination showed macroglossia. Laboratory findings showed a decrease in hemoglobin concentration $(10.1 \mathrm{~g} / \mathrm{dl})$; prolongation of prothrombin time (PT) expressed as an international normalized ratio (INR) of 1.13; and elevated cardiac markers (troponin I $0.131 \mathrm{ng} / \mathrm{ml}$ [reference range: < $0.04 \mathrm{ng} / \mathrm{ml}$ ], NT-proBNT 2,739 pg/ml [reference range: $<222 \mathrm{pg} / \mathrm{ml}]$ ). Chest radiography was consistent with previous findings of cardiomegaly without active lung lesions. Sinus rhythm with atrial premature complexes and left ventricular hypertrophy were found in electrocardiogram. An echocardiogram showed increased left ventricle (LV) wall thickness, diastolic dysfunction (grade 2) with increased LV filling pressure, both atrial enlargement, and a moderate amount of pericardial effusion. The LV ejection fraction was $60.5 \%$ without regional wall motion abnormality. The right ventricle cavity size and systolic function were normal. These findings were an improvement compared to echocardiogram findings from 4 months previously. During consultations, cardiologists agreed that the patient's cardiac condition had improved and that she would be able to undergo the operation. Nerve conduction study results were suggestive of amyloidosis-associated polyneuropathy with abnormal electrophysiology. Autonomic nerve function tests showed severe sudomotor, adrenergic, cardiovagal autonomic dysfunction. After consulting with cardiologists, we decided to proceed without inserting a temporary pacemaker. Instead, an external patch and defibrillator were prepared in the operating room for possible intraoperative cardiac complications such as severe atrioventricular block. Prior to induction, trans-esophageal echocardiography (TEE) was set up in the operating room to ensure that it was ready to use if needed; however, it was not inserted to prevent complications such as varix bleeding.

The arterial line was acquired with administration of local anesthesia before induction in order to monitor arterial blood pressure during induction. Induction was successfully done with $16 \mathrm{mg}$ of etomidate, $8 \mathrm{mg}$ of vecuronium, and isoflurane. Intubation was easily performed with a Macintosh blade \#3. Macroglossia with nodules due to amyloidosis was noted, but the vocal cord was spared for infil- tration. An additional arterial line at the right femoral artery was acquired. The central venous line was also placed in the right femoral and right internal jugular veins. A multifunction pulmonary artery catheter was inserted to monitor cardiac output, pulmonary arterial pressure, pulmonary artery occlusion pressure, and mixed venous oxygen saturation. During these procedures, the patient's heart rate did not change in response to the stimuli, such as intubation or line insertion. Instead, the heart rate slowly but continuously decreased to 48 beats $/ \mathrm{min}$. Isoproterenol $(1 \mu \mathrm{g} / \mathrm{min})$ was administered and the heart rate increased to 80 beats/ $\mathrm{min}$. Isoproterenol was tapered to $0.5 \mu \mathrm{g} / \mathrm{min}$ and the infusion rate was maintained. Remifentanil and norepinephrine were used appropriately to control blood pressure, and vecuronium was infused continuously to ensure adequate neuromuscular blockade.

Blood flow to and from the left lobe was kept intact to minimize the changes in hemodynamics throughout the procedures. Post-reperfusion syndrome was noted with a decrease in mean arterial pressure from 75 to $36 \mathrm{mmHg}$ without a change in heart rate (92 beats/min). Pulmonary arterial pressure decreased from 18 to $13 \mathrm{mmHg}$. Phenylephrine $(50 \mu \mathrm{g})$ was administered and the continuous infusion rate of norepinephrine was increased; autologous blood from Cell Saver was transfused to normalize the blood pressure. Hemodynamic parameters remained stable after the intervention with a vasopressor; thus, TEE was not used throughout the procedure.

The total urine output was $685 \mathrm{ml}$, and the estimated lost $\mathrm{RBC}$ mass was $641 \mathrm{ml}$ [7]. One unit of pre-leukocyte-reduced RBC (PLRBC) and $471 \mathrm{ml}$ of autologous blood from Cell Saver was transfused and the operation commenced uneventfully. The patient was transferred to the intensive care unit (ICU) while intubated after removal of the pulmonary artery catheter. Continuous isoproterenol infusion was maintained during the transfer to the ICU. During the transfer from the operation table to the hospital bed, the external patch was incidentally removed but was not reattached because the patient's vital signs were stable.

Postoperative laboratory values were as follows: hemoglobin $9.6 \mathrm{~g} / \mathrm{dl}$, platelet counts $114 \times 10^{3} / \mu \mathrm{l}$, PT INR 1.62, and fibrinogen $180 \mathrm{mg} / \mathrm{dl}$. Five hours later, continuous infusion of vasopressin $(0.03 \mathrm{unit} / \mathrm{min})$ was added to maintain blood pressure, and isoproterenol infusion was stopped due to sinus tachycardia (120 beats/min). On the 1st POD, the patient showed stable vital signs and alert mental status thus, the patient was weaned off the ventila- 
tor and the endotracheal tube was removed. The patient remained alert and vital signs were stable after extubation. However, after $1 \mathrm{~h}$, the patient suddenly complained of chest discomfort, and her mental status changed to stupor. Vital signs showed a blood pressure of 66/19(30) $\mathrm{mmHg}$, heart rate of 120 beats/min, and $\mathrm{SpO}_{2}$ of $94 \%$; intubation was performed immediately. One liter of Plasma Solution $\mathrm{A}^{\circledR}$ and 1 unit of PLRBC were administered rapidly, and a continuous infusion of norepinephrine $(0.6 \mu \mathrm{g} / \mathrm{kg} / \mathrm{min})$ was started. Two hours and eight minutes after intubation, ventricular tachycardia was observed on electrocardiogram. Cardioversion with $200 \mathrm{~J}$ was performed twice, and amiodarone (150 mg) was administered intravenously as a loading dose. A blood pressure of 81/51(59) mmHg with a heart rate of 130 beats/min was achieved after the intervention. Three units of fresh frozen plasma and six units of cryoprecipitate were transfused. However, after $1 \mathrm{~h}$ and 3 min of cardioversion, pulseless electrical activity (PEA) occurred. Cardiopulmonary resuscitation (CPR) was performed with a total bolus injection of epinephrine (14 mg) and recovery of spontaneous circulation (ROSC) was achieved; a continuous infusion of dobutamine $(5 \mu \mathrm{g} / \mathrm{kg} /$ min) was added. Eighteen minutes after the first ROSC, PEA was checked again and we performed CPR for a second time. Ten minutes after the second CPR session, ROSC was achieved again. However, 13 min after the second ROSC, PEA occurred again. CPR was started for a third time, but she died $4 \mathrm{~h}$ and $37 \mathrm{~min}$ after intubation.

\section{Case 3. Second recipient}

A 64-year-old man agreed to undergo domino LT. He was diagnosed with liver cirrhosis due to hepatitis B virus infection and had undergone multiple transarterial chemo-embolization due to hepatocellular carcinoma. Without a living donor and with a low model for end-stage liver disease score of 9, he was not eligible for LDLT or deceased donor LT (DDLT). He agreed to undergo domino LT with a liver graft from a FAP patient.

The patient was on medication for hypertension and diabetes mellitus II. Laboratory findings were consistent with cirrhotic liver and chronic illness showing a hemoglobin of $8.6 \mathrm{~g} / \mathrm{dl}$, platelet count of $89 \times 10^{3} / \mu \mathrm{l}$, and a prolonged prothrombin time (INR) of 1.34. All other test results were within the normal range. Upper and lower gastrointestinal endoscopy was performed and no active bleeding lesions were found, but esophageal and gastric varices were found.
Induction was performed with thiopental ( $325 \mathrm{mg}$ ), atracurium (40 mg), and sevoflurane. Intubation was performed with ease and without complications. Arterial and central lines were placed with a multifunction pulmonary artery catheter. Anesthesia was maintained with isoflurane and continuous infusion of atracurium. The liver was uneventfully extracted. At the start of the anhepatic phase, Hepabig 10,000 IU mixed with $200 \mathrm{ml}$ of 5\% dextrose solution was infused. Dopamine and norepinephrine were given at rates of $3 \mu \mathrm{g} / \mathrm{kg} / \mathrm{min}$ and $0.05 \mu \mathrm{g} / \mathrm{kg} / \mathrm{min}$, respectively, to maintain blood pressure. The total urine output was $1,100 \mathrm{ml}$, and the estimated lost RBC mass was $874 \mathrm{ml}$. Three packs of PLRBC and $100 \mathrm{ml}$ of blood from Cell Saver were transfused. The patient was transferred to the ICU while still intubated. He was moved to the general ward on the 6th POD without any complications and was discharged 2 weeks later.

\section{DISCUSSION}

FAP is the hereditary form of TTR-related amyloidosis which is caused by mutations in the TTR gene, which alters the secondary and tertiary structure to alter metabolism and amyloid fibril formation [2]. LT is the only treatment option that can stop its progression; however, it presents various challenges for anesthetic management of these patients. Meanwhile, explanted liver from FAP patients is fully functional, except for the production of the mutated amyloids, which becomes a clinical problem in the years following the transplant to a non-FAP patient. Thus, as in our case, it can be a possible treatment option for those who may not be candidates for either LDLT or DDLT. Since domino LT is possible, with the presence of a structurally and functionally normal liver of FAP patients, FAP patients are important for a successful operation.

Various intraoperative anesthetic management techniques should be considered for patients with FAP. Peripheral vasodilation, cardiac autonomic denervation, and restrictive cardiomyopathy secondary to amyloid infiltration contribute to hypotension $[8,9]$. Intraoperative TEE is a useful modality to differentiate the cause of hypotension [10]. Although TEE was prepared in case it was needed to identify the cause of hypotension during the procedure, there was no incidence of unstable hemodynamic changes that were not already expected during the LDLT procedures. There was an event of PRS; however, it was quickly corrected with vasopressor interventions. If there was an 
event that was not expected during regular LDLT procedures, TEE could provide additional information that would be useful for differentiation of hypotension.

The patient had cardiac denervation, which was presented as bradycardia in this case. Therefore, the patient did not manifest dramatic increases in heart rate or blood pressure, despite preparing the patient for the operation (e.g., intubation, position change). Cardiac autonomic denervation in FAP does not alter cardiac beta-receptor responsiveness to catecholamine, thus isoproterenol is used to increase heart rate while atropine may be ineffective [8].

Isoflurane is known to decrease arterial pressure mainly by reducing systemic vascular resistance with minimal effect on cardiac output compared with other volatile anesthetics [11]. Use of isoflurane for patients with FAP for resolution of sinus dysrhythmia has also been reported, thus indicating that it is a good choice for maintaining anesthesia [12]. Researchers speculate that isoflurane decreases the rate of phase 4 depolarization of sinoatrial node cells [13].

There are discrepancies in implementation of preoperative pacemaker insertion in FAP patients with cardiac involvement who are undergoing LT [14]. After in-depth discussion with cardiologists and surgeons, we decided to omit temporary pacemaker insertion and proceeded with the procedure using an external patch. It was thought to give the same benefit as the pacemaker without the patient having to go through another meticulous procedure. Implementation of a pacemaker could provide stable rhythm control compared to an external patch. However, undergoing the procedure may burden the FAP patient. In addition, the placed wire can interfere with the pulmonary arterial catheter introduced during induction, causing complications such as arrhythmia. If the pacemaker wire was introduced via femoral access, the position could be altered during inferior vena cava clamping during the operation.

Despite timely CPR management in the ICU, the patient was not resuscitated. Autonomic denervation of the FAP patient's heart makes it resistant to inotropic and chronotropic actions that we routinely use in the cycles of CPR. It would have been more beneficial if isoproterenol was used. There are reports of sudden unexpected death of patients with cardiac amyloidosis which may be a result of arrhythmias or disturbances of conduction [15]. The patient's heart was functionally at risk for conduction failure; it would thus have been helpful if the external patch or pacemaker was in place post-operatively.
Notably, this is the first case of domino LT in Korea and anesthetic management was successfully performed without complications during the operation. However, there are still challenges in managing patients with FAP post-operatively, which would dramatically alter the course of the patient.

\section{CONFLICTS OF INTEREST}

No potential conflict of interest relevant to this article was reported.

\section{AUTHOR CONTRIBUTIONS}

Conceptualization: Soo Joo Choi, Gaab Soo Kim. Data curation: Sungrok Cha, Jiwon Kim. Formal analysis: Sungrok Cha, Gaab Soo Kim. Methodology: Gaab Soo Kim. Writing - original draft: Sungrok Cha. Writing - review \& editing: Sungrok Cha. Investigation: Sungrok Cha, Jiwon Kim. Resources: Jiwon Kim. Supervision: Soo Joo Choi, Gaab Soo Kim. Validation: Soo Joo Choi, Gaab Soo Kim.

\section{ORCID}

Sungrok Cha, https://orcid.org/0000-0002-3140-1822

Jiwon Kim, https://orcid.org/0000-0001-8198-1274

Soo Joo Choi, https://orcid.org/0000-0002-5212-2451

Gaab Soo Kim, https://orcid.org/0000-0002-9383-2652

\section{REFERENCES}

1. Andrade C. A peculiar form of peripheral neuropathy; familiar atypical generalized amyloidosis with special involvement of the peripheral nerves. Brain 1952; 75: 408-27.

2. Benson MD. Liver transplantation and transthyretin amyloidosis. Muscle Nerve 2013; 47: 157-62.

3. Sekijima Y. Transthyretin (ATTR) amyloidosis: clinical spectrum, molecular pathogenesis and disease-modifying treatments. J Neurol Neurosurg Psychiatry 2015; 86: 1036-43.

4. Adams D, Samuel D, Goulon-Goeau C, Nakazato M, Costa PM, Feray C, et al. The course and prognostic factors of familial amyloid polyneuropathy after liver transplantation. Brain 2000; 123(Pt 7): 1495-504.

5. Furtado A, Tomé L, Oliveira FJ, Furtado E, Viana J, Perdigoto R. Sequential liver transplantation. Transplant Proc 1997; 29: 4678.

6. Stangou AJ, Heaton ND, Hawkins PN. Transmission of systemic 
transthyretin amyloidosis by means of domino liver transplantation. N Engl J Med 2005; 352: 2356.

7. Bang SR, Ahn HJ, Kim GS, Yang M, Gwak MS, Ko JS, et al. Predictors of high intraoperative blood loss derived by simple and objective method in adult living donor liver transplantation. Transplant Proc 2010; 42: 4148-50.

8. Delahaye N, Dinanian S, Slama MS, Mzabi H, Samuel D, Adams D, et al. Cardiac sympathetic denervation in familial amyloid polyneuropathy assessed by iodine-123 metaiodobenzylguanidine scintigraphy and heart rate variability. Eur J Nucl Med 1999; 26: 416-24.

9. Delahaye N, Le Guludec D, Dinanian S, Delforge J, Slama MS, Sarda L, et al. Myocardial muscarinic receptor upregulation and normal response to isoproterenol in denervated hearts by familial amyloid polyneuropathy. Circulation 2001; 104: 29116.

10. Neelakanta G, Mahajan A, Antin C. Systemic vasodilation is a predominant cause of hypotension in a patient with familial amyloid polyneuropathy during liver transplantation. J Clin Anesth 2005; 17: 202-4.

11. Eger EI 2nd. Isoflurane: a review. Anesthesiology 1981; 55: 55976.

12. Castro Tavares J, Maciel L. Anaesthetic management of a patient with familial amyloid polyneuropathy of the Portuguese type. Can J Anaesth 1989; 36: 209-11.

13. Bosnjak ZJ, Kampine JP. Effects of halothane, enflurane, and isoflurane on the SA node. Anesthesiology 1983; 58: 314-21.

14. Milner J, Teixeira RN, Marinho AV, Silva N, Calretas S, Ferrão J, et al. Pacemaker implantation in familial amyloid polyneuropathy: when and for whom? J Interv Card Electrophysiol 2019; 55: 207-11.

15. Eriksson P, Karp K, Bjerle P, Olofsson BO. Disturbances of cardiac rhythm and conduction in familial amyloidosis with polyneuropathy. Br Heart J 1984; 51: 658-62. 